many found the process of finger-prick blood sampling extremely challenging and this contributed to concerns that test results may be inaccurate. A minority of participants also missed the opportunity clinic-based testing offered to discuss symptoms or concerns with staff. Participants overwhelmingly found the process of receiving test results by SMS acceptable and preferable to alternatives.

Conclusions Internet-based testing is viewed positively by most users but uptake may be improved if providers emphasise the privacy and convenience it offers, as well as the accuracy of self-sampling. Providers should also consider measures to address user concerns around blood sampling and the lack of specialist advice. Further research involving larger numbers of users, and focusing specifically on populations with low uptake of internet-based testing, would be worthwhile.

\section{P415 ESTABLISHMENT OF SPECIAL TREATMENT CLINIC FOR SEXUALLY TRANSMITTED INFECTIONS IN GOMBE NIGERIA: REALITIES AND PROSPECTS}

M Manga*, H Farouk, A Mohammed, U Hassan, M Ibrahim. Gombe State University and Federal Teaching Hospital, Gombe, Nigeria

10.1136/sextrans-2021-sti.440

Background/Purpose The medical, socio-cultural and religious perspectives in managing sexually transmitted infections (STIs) have expanded and made more critical the need for multidisciplinary/'one health' approach to tackling this global menace. Expertise, convenience and efficiency have remained the major considerations in selecting approaches and avenues for diagnosis and treatment of STIs. The understanding and acceptability of patients and other healthcare workers is cardinal in the success of any STI clinic. We present our experiences/observations of establishing and maintaining a new STI clinic in a low resource setting.

Approach The Federal Teaching Hospital Gombe (FTHG) Special Treatment Clinic (STC), was established in 2015 with sole aim of providing quality care for patients with genital tract infections from within and outside the hospital. Specialist consultations, confirmatory laboratory investigations and record keeping are all brought together under one roof for convenience and efficiency. Other requests for genital tract microbiological investigations from all parts of the hospital are also pooled to the clinic for both specimen collection and immediate processing.

Outcomes/Impact The clinic has now provided avenue of combining both clinical consultation and laboratory diagnosis within the limited available resources. It has gained the confidence of patients from the community and healthcare workers within the hospital. Majority (60.1) of patients seen at the clinic were self-referrals followed by those referred from the general outpatient clinics of the hospital (27.0\%) and a few (3.8\%) from outside FTHG. This has provided the most needed alternative to a growing menace of quackery in managing STIs by unqualified traditionalists.

Innovation and Significance Improvement in confidentiality and efficiency has continued to make our clinic most preferred for consultations and treatment of STIs. The near obliteration of delays in specimen transportation with their immediate processing has made it possible to now identify pathogens such as Neisseria gonorrheae, Trichomonas vaginalis.

\section{P416 SCREENING RATES AND FOLLOW-UP OF CHLAMYDIA TRACHOMATIS AND NEISSERIA GONORRHOEAE INFECTIONS DURING PREGNANCY}

${ }^{1} \mathrm{R}$ Thibeault* , ${ }^{1} \mathrm{R}$ Escobar Careaga, ${ }^{2} \mathrm{C}$ Lavallée, ${ }^{2} \mathrm{~A}$ Labbé, ${ }^{3} \mathrm{G}$ Roy, ${ }^{3} \mathrm{C}$ Fortin. ${ }^{1}$ University of Montreal, Montréal, Canada; ${ }^{2}$ Hôpital Maisonneuve-Rosemont, CIUSSS de I'Est-de-I'Île-deMontréal, Montréal, Canada; ${ }^{3}$ Centre hospitalier de I'Université de Montréal, Montréal, Canada

\subsection{6/sextrans-2021-sti.441}

Introduction Doubts concerning the efficacity of prophylactic ocular topic antibiotics on newborns for prevention of ophtalmia neonatorum $(\mathrm{ON})$ and its decreasing incidence lead the Canadian Pediatric Society to no longer recommend its systematic use. They rather recommend that prevention of $\mathrm{ON}$ should focus on systematic prenatal screening of Chlamydia trachomatis (CT) and Neisseria gonorrhoeae (NG) and treatment of infected women and their partners. This study's primary endpoint was to evaluate compliance with Quebec's provincial pregnancy screening, test of cure (TOC) and rescreening guidelines.

Methods Charts of all women who delivered at the Centre hospitalier de l'Université de Montréal, a tertiary care hospital in Montreal, between April 1st, 2018 and March 31st, 2019 were reviewed to assess demographic, clinical and laboratory data.

Results Among 2557 women, 2481 (97.0\%) were screened for $\mathrm{CT} / \mathrm{NG}$ at least once during pregnancy. For 2278 women (89.1\%) a laboratory report was retrievable for analysis. CT infection was detected in 20/2278 women $(0,88 \%)$ whose mean age was 33 years old (range from 18 to 39). Treatment was appropriate according to guidelines in 18 (90\%), a test of cure (TOC) was performed in 18 (90\%), and re-screening between 3 and 6 months after diagnosis was done in 14 women (70\%). Treatment of sexual partners was documented for 16 women (80\%). The only NG infection detected $(0,04 \%)$ was wrongly interpreted as a false positive; no treatment was administered.

Conclusion The proportion of pregnant women with at least one available CT/NG test result compares favorably to screening rates in other Canadian studies. However, the proportion of CT infected women for whom a TOC and re-screening was performed needs to be improved. The interpretation of positive NG tests in pregnant women and documentation of sexual partners management of infected women also needs improvement.

\section{P417 RISK OF CHLAMYDIA AND GONORRHEA AMONG YOUNG AFRICAN AMERICAN WOMEN WITH PERSISTENT AND EPISODIC BACTERIAL VAGINOSIS}

${ }^{1,2} \mathrm{M}$ Coudray*, ${ }^{2} \mathrm{D}$ Sheehan, ${ }^{2} \mathrm{~T}$ Li, ${ }^{3} \mathrm{R}$ Cook, ${ }^{4} \mathrm{~J}$ Schwebke, ${ }^{5} \mathrm{P}$ Madhivanan. ${ }^{1}$ University of Central Florida, Orlando, USA; ${ }^{2}$ Florida International University, Miami, USA; ${ }^{3}$ University of Florida, Gainesville, USA; ${ }^{4}$ University of Alabama a Birmingham, Birmingham, USA; ${ }^{5}$ University of Arizona, Tuscon, USA

\subsection{6/sextrans-2021-sti.442}

This study aimed to assess the influence of episodic and persistent bacterial vaginosis (BV) on incident Chlamydia trachomatis (CT) and Neisseria gonorrhoeae (NG) infection among young African American women in the US. 
Data from 428 women, were included in this secondary data analysis. All women were asymptomatic for BV at baseline and tested for BV every two months. Persistent cases of $\mathrm{BV}$ were positive for BV at month two, four and six. Women who were negative for BV at month two, four and six were classified as no BV. All other cases were defined as episodic BV. Incident STI was defined as any new case of CT or NG at month eight. Factors associated with STI acquisition were assessed using Binary Logistic Regression. The final model was adjusted for age, education, women who have sex with women and BV status.

Most women were $\leq 21$ years $(55.8 \%)$ and completed some post high school/GED education (50.9\%). There were 179 (41.8\%) women with persistent BV, 204 (47.7\%) women with episodic BV and 45 (10.5\%) women with no BV. At month eight $8.6 \%$ of women tested positive for an STI. Bivariate analysis demonstrated a significant association between women with no BV and STI acquisition $(\mathrm{p}=0.02)$. Women with no BV did not acquire an STI, 7.3\% of women with persistent BV acquired and STI and 11.7\% of women with episodic BV acquired an STI. Women with high school or more than high school education compared to women with less than high school education had decreased odds of developing an STI (Adjusted Odds Ratio (adjOR): 0.38; 95\% CI: 0.15-0.92; and adjOR: 0.31 ; 95\%CI: $0.13-0.73$ ).

At least a high school education was associated with increased odds of STI acquisition compared to less than high school education. STI acquisition was only observed among women with BV.

\section{P418 CORRELATES OF IMMUNE PROTECTION IN THE RABBIT MODEL OF SYPHILIS VACCINATION}

T Reid*, A Haynes, E Romeis, C Godornes, V Campbell, D Koelle, L Giacani. University of Washington, Seattle, USA

\subsection{6/sextrans-2021-sti.443}

Background An effective syphilis vaccine will be integral to efforts to eradicate this disease. Promising vaccine candidates are surface antigens of the syphilis spirochete, Treponema pallidum subsp. pallidum ( $T$. pallidum). These antigens can be targeted by vaccination-induced opsonic antibodies and mediate pathogen immune clearance. Defining correlates of protection will aid in the identification of the best vaccine candidates. Here, we sought to investigate whether immunization with variants of the $\mathrm{T}$. pallidum Repeat $\mathrm{C}$ (TprC) protein and the conserved NH2-terminus of the TprK induced protection and whether splenocyte proliferation and IFN- $\gamma$ production correlated with protection.

Methods Rabbits were immunized with either a cocktail of three recombinant, full-length $\mathrm{TprC}$ variants, or the NH2-terminus of the TprK protein with a RIBI-like adjuvant. Animals were challenged with $\mathrm{T}$. pallidum intradermally (10 sites; $10^{\wedge} 5$ bacteria/site). Treponemal burden and progression to ulceration were monitored. To assess for immunogen-specific splenocytes, pools of synthetic peptides corresponding to each immunogen were used to stimulate splenocytes collected exvivo in proliferation assays. Supernatants from stimulated splenocytes were used to quantify IFN- $\gamma$ responses by ELISA.

Results Immunizations protected animals significantly albeit not completely. At day 35 post-challenge only $14.1 \%$ and $15.5 \%$ of lesions ulcerated in immunized rabbits compared to the
$95 \%$ of lesions in unimmunized rabbits. At day 21, there was a $99.3 \%$ and $98.7 \%$ reduction in treponemal burden averaged across all challenge sites in TprC- and TprK-immunized rabbits compared to unimmunized animals, respectively. Lymphocyte proliferation and IFN- $\gamma$ production correlated to reduction in both percent of ulcerated lesions and treponemal burden.

Conclusions Lymphocyte proliferation and IFN- $\gamma$ release assays may serve as surrogates to assess for antigen-specific T-cell responses. TprK and $\mathrm{TprC}$ immunizations are able to stimulate cellular immunity in a $\mathrm{TH} 2$ environment, which is key to development of an effective syphilis vaccine.

\section{P420 RHEIN INHIBITS CHLAMYDIA TRACHOMATIS INFECTION BY REGULATING PATHOGEN-HOST CELL INTERACTIONS}

X Yu*, W Chen, Q Xu, X Su, Y Xue, H Zheng. Dermatology Hospital, Southern Medical University, Guangzhou, China

\subsection{6/sextrans-2021-sti.444}

Background The global incidence of genital Chlamydia trachomatis (C. trachomatis) infection increased rapidly as the primary available treatment of $\mathrm{C}$. trachomatis infection being the use of antibiotics; however, the development of antibiotics resistant stains and other treatment failures are often observed in patients. Consequently, novel therapeutics are urgently required. Rhein is a monomer derivative of anthraquinone compounds with an anti-infection activity. This study investigated the effects of rhein on treating C. trachomatis infection.

Methods The cytotoxicity of rhein was examined by Cell Counting Kit (CCK) 8 assay. Rhein's inhibitory effect on C. trachomatis infection was detected by immunofluorescent staining, electron microscopy, and progeny infection titer assay. The protein levels of p-RSK, p-ERK, RSK, ERK, cHSP60 were detected by immunoblotting assays. Lastly, C. trachomatis infected mouse model was used to determine the in vivo antiinfection effect of rhein.

Results Rhein showed significant inhibitory effects on the growth of C. trachomatis in multiple serovars of C. trachomatis, including $\mathrm{D}, \mathrm{E}, \mathrm{F}$, and $\mathrm{L} 1$, and in various host cells, including HeLa, McCoy, and Vero. Rhein could not directly inactivate C. trachomatis but could inhibit the growth of C. trachomatis by regulating pathogen-host cell interactions. Host p-ERK and p-RSK were both down-regulated in the presence of rhein at $36 \mathrm{~h}$ and $48 \mathrm{~h}$ post-infection. Combined with azithromycin, the inhibitory effect of rehin was synergistic both in vitro and in vivo.

Conclusion We found rhein inhibited C. trachomatis infection regulating pathogen-host cell interactions. Together these findings suggest that rhein could be developed for the treatment of C. trachomatis infections.

\section{P423 TAKING REAL ISSUES TO VIRTUAL CONFERENCES: THE TRAINING OF YKP TO DISCUSS SOCIAL VULNERABILITY MEASURES IN THE COVID-19 CONTEXT}

D Agostinho Calixto*, N Fagundes Correia, C Moreira Aló, C Bernardes Sousa. Ministry of Health of Brazil

10.1136/sextrans-2021-sti.445 